Study of different h-indices for groups of authors

Peer-reviewed author version

EGGHE, Leo \& RAO, Ravichandra (2008) Study of different h-indices for groups of authors. In: JOURNAL OF THE AMERICAN SOCIETY FOR INFORMATION

SCIENCE AND TECHNOLOGY, 59(8). p. 1276-1281.

DOI: 10.1002/asi.20809

Handle: http://hdl.handle.net/1942/7918 


\section{Study of different h-indices \\ for groups of authors}

by

L. Egghe ${ }^{(1,2,4)}$

and

I.K. Ravichandra Rao ${ }^{(1,3)}$

Universiteit Hasselt (UHasselt), Campus Diepenbeek, Agoralaan, B-3590 Diepenbeek, Belgium $^{(1)}$

Universiteit Antwerpen (UA), Campus Drie Eiken, Universiteitsplein 1, B-2610 Wilrijk, Belgium $^{(2)}$

Indian Statistical Institute (ISI), $8^{\text {th }}$ Mile, Mysore Road, R.V. College P.O., Bangalore560059, India $^{(3)}$

\section{ABSTRACT}

In this paper, for any group of authors, we define three different h-indices. First there is the successive h-index $h_{2}$ based on the ranked list of authors and their h-indices $h_{1}$ as defined by Schubert. Next there is the h-index $h_{P}$ based on the ranked list of authors and their number of publications. Finally there is the $\mathrm{h}$-index $\mathrm{h}_{\mathrm{C}}$ based on the ranked list of authors and their number of citations.

We present formulae for these three indices in Lotkaian informetrics from which it also follows that $h_{2}<h_{P}<h_{C}$. We give a concrete example of a group of 167 authors on the topic

\footnotetext{
${ }^{4}$ Corresponding author.
}

Key words and phrases: h-index, Hirsch, group of authors.

Acknowledgement: The second named author is grateful to the Universiteit Hasselt for financial support during the time he was a visiting professor. 
"optical flow estimation". Besides these three h-indices we also calculate the two-by-two Spearman rank correlation coefficient and prove that these rankings are significantly related.

\section{Introduction}

The h-index (or Hirsch index) of an author has been introduced in Hirsch (2005) as follows. If we rank the publications of an author in decreasing order of the number of citations that these articles received then this author's $\mathrm{h}$-index $\mathrm{h}$ is the largest rank such that all the articles on rank $1,2, \ldots, \mathrm{h}$ have $\mathrm{h}$ or more citations.

The h-index, introduced only 2 years ago, has become a real hype in and even outside informetrics: Ball (2005, 2007), Bornmann and Daniel (2005, 2007a), Braun, Glänzel and Schubert $(2005,2006)$ (introducing the h-index for journals, yielding a new journal indicator to be preferred above the impact factor - see Miller (2006)), Egghe and Rousseau (2006), Glänzel (2006a,b), Popov (2005), van Raan (2006), Bar-Ilan (2006), Rousseau (2007), Burrell (2007a,b), Glänzel and Persson (2005), Egghe (2007c), Saad (2006), Oppenheim (2007), Hirsch (2007), Barendse (2007), Wan, Hua and Rousseau (2007), Rao and Rousseau (2007), Vinkler (2007), Vanclay (2007) and see also the papers in the special issue on the Hirsch index in Journal of Informetrics 1(3), 2007: Schubert and Glänzel (2007), Beirlant, Glänzel, Carbonez and Leemans (2007), Costas and Bordons (2007) and Bornmann and Daniel (2007b).

Banks (2006) introduces the interesting notion of the h-index for topics and compounds - see also Egghe and Rao (2007) and the STIMULATE6 Group (2007). Let us, finally note that both the Web of Science and Scopus offer the h-index in their databases (remarkably quick after its introduction in 2005!).

Schubert and Prathap (independently and almost simultaneously) introduced the interesting idea of "successive h-indices" (see Schubert (2007), Prathap (2006) - see also http://11011110.livejournal.com/10507.html and www.cs.utah.edu/ shirley/hindex/ and Egghe (2007b). Denote the h-index of an author in a group of authors by $h_{1}$. If we rank these authors in decreasing order of their h-index $h_{1}$ we can then apply the definition of the $h$-index 
to this ranked list and obtain the h-index $h_{2}$ of this group of authors (e.g. an institute, hereby giving a visibility score of this institute). This is the first h-index for a group of authors that we will use in this paper. Now we introduce two other ones for the same group of authors.

h-indices can be calculated on any system of sources and items ( a so-called information production process (IPP)) where the sources are ranked in decreasing order of their number of items. As sources we will always take the group of authors under consideration (as we did also above). For our next h-index of this group we rank these authors in decreasing order of their number of publications. The h-index of this ranked group will be denoted by $h_{p}$.

For our third h-index we rank the same group of authors in decreasing order of their total number of citations that where received by the publications of these authors. The h-index of this ranked group will be denoted by $\mathrm{h}_{\mathrm{C}}$.

Apart from the paper Liu and Rousseau (2007), where the h-index was applied to library circulation data, the present paper - we think - is the first to also apply the h-index to noncitation data.

In the next section we will present models for these three indices based on results in Lotkaian informetrics. We will derive from these models that $\mathrm{h}_{2}<\mathrm{h}_{\mathrm{P}}<\mathrm{h}_{\mathrm{C}}$.

In the third section a concrete example will be given: 167 authors in the field "optical flow estimation". We find in this case: $\mathrm{h}_{2}=10, \mathrm{~h}_{\mathrm{P}}=20$ and $\mathrm{h}_{\mathrm{C}}=67$. We also calculate the Spearman rank correlation coefficient for each couple of author rankings and show that the ranks correlate significantly.

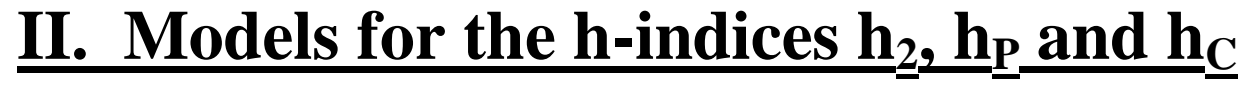

Let us start with the simplest model: the one for $h_{\mathrm{p}}$. 


\section{$\underline{\text { II.1 Model for } h_{\underline{P}}}$}

Let there be $\mathrm{T}$ authors and suppose that their author-publication IPP is ruled by a Lotkaian law of the form

$$
f(j)=\frac{C}{j^{\alpha_{1}}}
$$

where $\mathrm{C}>0, \alpha_{1}>1, \mathrm{j} \hat{I}[1,+¥[: \mathrm{f}(\mathrm{j})$ denotes the density of the authors with publication density $\mathrm{j}$ - see Egghe (2005), Chapter II. The requirement $\alpha_{1}>1$ makes sure that

$$
T=\grave{O}_{1}^{*} f(j) d j=\frac{C}{\alpha_{1}-1}
$$

is finite. It is generally true that the Lotka exponent $\alpha_{1}$ is larger than 1 in practice.

In Egghe and Rousseau (2006) we showed that in such Lotkaian systems, the h-index is given by

$$
\mathrm{h}_{\mathrm{P}}=\mathrm{T}^{\frac{1}{\alpha_{1}}}
$$

So this result follows from Egghe and Rousseau (2006) in a straightforward way.

\section{$\underline{\text { II.2 Model for } \mathbf{h}_{\mathrm{C}}}$}

Of course, as for $\mathrm{h}_{\mathrm{P}}$, we can here make the same argument, supposing a Lotka law as in (1) but with another value for $\alpha_{1}$, say $\delta$ and we then have, by Egghe and Rousseau (2006):

$$
\mathrm{h}_{\mathrm{C}}=\mathrm{T}^{\frac{1}{\delta}}
$$

But we can do better than that: we can present a formula for $\delta$ in function of $\alpha_{1}$ and a "positive reinforcement" parameter $\beta$, to be explained further on. Indeed, the situation described in this subsection is a typical case of a positive reinforcement of the situation described in the previous subsection - cf. Egghe (2005), Chapter III: the author-publications 
relation is composed with the publications-citations relation yielding the author-citations relation. It is proved in Theorem III.1.3.1.3 (page 164-167) that this yields a positive reinforcement of the item densities: $\mathrm{j}$ in (1) is transformed into $\varphi(j)$ where $\varphi(j)^{3} \mathrm{j}$ for all $\mathrm{j}^{3} 1$ since we only consider publications with at least 1 citation for the calculation of the $\mathrm{h}$ index $h_{C}$.

As we did in Section III.2 of Egghe (2005) we propose a power law for the function $\varphi$ :

$$
\varphi(j)=B j^{\beta}
$$

where $B^{3} 1$ and $\beta>1$ since we explained above that $\varphi(j)^{3} \quad j$ is a requirement for all $j^{3} 1$ (with $0<\beta<1$ we have that $\varphi(j)<j$ from some $j$ on ( $\mathrm{j}$ large enough) and $\beta=1$ is not considered since the publication-citation relation is a strictly positive reinforcement!).

We now invoke Corollary III.2.1.1 in Egghe (2005) proving that our authors-citations relation is also Lotkaian but with $\mathrm{f}$ replaced by $\left(\mathrm{j}_{1}=: \varphi(\mathrm{j})\right)$

$$
f^{*}\left(j_{1}\right)=\frac{D}{j_{1}^{\delta}}
$$

where $\mathrm{D}>0, \mathrm{j}_{1}{ }^{3} \mathrm{~B}^{3} 1$ and with

$$
\delta=\frac{\alpha_{1}+\beta-1}{\beta}
$$

Since now we have that $\mathrm{j}_{1}{ }^{3} \mathrm{~B}$ we have to use the extension of the result proved in Egghe and Rousseau (2006) on the h-index. This extension was proved in Egghe (2007a): we now have

$$
\mathrm{h}_{\mathrm{C}}=\mathrm{B}^{\frac{\delta-1}{\delta}} \mathrm{T}^{\frac{1}{\delta}}
$$

(since the authors = sources remain the same we have taken $\psi=1$ in Egghe (2007a)) 
Readers who are not familiar with this extension can simply take $B=1$ and apply the wellknown result in Egghe and Rousseau (2006):

$$
\mathrm{h}_{\mathrm{C}}=\mathrm{T}^{\frac{1}{\delta}}
$$

with $\delta$ as in (7). The reader will agree that (8) and (9) shed more light on $\mathrm{h}_{\mathrm{C}}$ than (4) because we have the knowledge of (7). So we have the following theorem:

Theorem 1: In the notation above, we have (take $\mathrm{B}=1$ ):

$$
\mathrm{h}_{\mathrm{C}}=\mathrm{T}^{\frac{\beta}{\alpha_{1}+\beta-1}}
$$

where $\alpha_{1}>1$ is the same as in (3) and where $\beta>1$ is as in (5).

This has the following corollary:

\section{Corollary 1:}

$$
\mathrm{h}_{\mathrm{C}}>\mathrm{h}_{\mathrm{P}}
$$

Proof: This follows from the fact that

$$
\frac{\beta}{\alpha_{1}+\beta-1}>\frac{1}{\alpha_{1}}
$$

(since $\beta>1$ and $\alpha_{1}>1$ ) and by (3) and (10).

Of course, in practise the above corollary is trivial since $\varphi(\mathrm{j})^{3} \mathrm{j}$ enforces the item numbers in positively transformed table to be larger than the ones in the original table and hence, the hindex of the transformed table must be strictly larger than the one of the original table!

Finally, we study $h_{2}$. 


\section{$\underline{\text { II.3 Model for } \mathbf{h}_{2}}$}

In Egghe (2007b) we have modelled successive h-indices which we described in the introductory section. This means that, for a group of authors we assume that, per author, the publication-citation relation is ruled by a law of Lotka of the form

$$
\mathrm{F}(\mathrm{k})=\frac{\mathrm{E}}{\mathrm{k}^{\alpha_{2}}}
$$

where E>0 and $\alpha_{2}>1$. This expresses - per author - the density of the papers with a density of $\mathrm{k}$ citations received. Since we also assume that the group of authors is homogeneous (e.g. all authors are from the same field) we can, as a simplification, assume that $\alpha_{2}$ is the same for all authors.

Let there be $\mathrm{j}$ publications:

$$
\begin{gathered}
j=\grave{O}_{1}^{¥} F(k) d k \\
j=\frac{E}{\alpha_{2}-1}
\end{gathered}
$$

Hence, from Egghe and Rousseau (2006) we derive that the $h$-index $h_{1}$ is given by

$$
\mathrm{h}_{1}=\mathrm{j}^{\frac{1}{\alpha_{2}}}
$$

Hence, by (1), taking $\mathrm{j}$ as a publication density of an author, the author- $\mathrm{h}_{1}$ relation is ruled by a distribution which is equal, up to a constant, to (combine (1) and (14))

$$
\frac{1}{\mathrm{~h}^{\alpha_{1} \alpha_{2}}}
$$


The complete theory is presented in Egghe (2007b). Applying again Egghe and Rousseau (2006), assuming as in (1) that there are T authors, we have, by definition of $h_{2}$ that

$$
\mathrm{h}_{2}=\mathrm{T}^{\frac{1}{\alpha_{1} \alpha_{2}}}
$$

, based on (15). We have indicated the proof of the following theorem (see Egghe (2007b) for a complete proof):

Theorem 2: If we have (12) for the publication-citation relation and (1) for the authorpublication relation, then the successive h-index $h_{2}$ of the group of authors is given by

$$
\mathrm{h}_{2}=\mathrm{T}^{\frac{1}{\alpha_{1} \alpha_{2}}}
$$

where $\mathrm{T}$ is as in (3) or (10).

Corollary 2: In all cases

$$
\mathrm{h}_{2}<\mathrm{h}_{\mathrm{P}}<\mathrm{h}_{\mathrm{C}}
$$

Proof: That $h_{P}<h_{C}$ has already been proved in Corollary 1. But, by (16)

$$
\begin{aligned}
\mathrm{h}_{2} & =\mathrm{T}^{\frac{1}{\alpha_{1} \alpha_{2}}} \\
& <\mathrm{T}^{\frac{1}{\alpha_{1}}}=\mathrm{h}_{\mathrm{P}}
\end{aligned}
$$

since $\alpha_{2}>1$ and by (3).

This closes the theoretical part of this paper. 


\section{Application}

We apply these indices to a bibliography of 167 authors on the topic "optical flow estimation". The bibliography was compiled from a list of 252 references selected from the website http://itbl.biologie.huerlin.de/ wiskott/Bibliographics/flowestimation.htma=ml in 2006. For each of the 167 authors, the number of papers was collected. The number of citations received by each of the papers of every author was determined using the Web of Science and so, the total number of citations received by every author was determined.

Six authors received no citations (hence $\mathrm{h}=0$ ). One author (J.J. Koenderink) had a maximum of $\mathrm{h}=25$. The data on the authorranks are shown in the appendix. We have obtained the following results.

$$
\mathrm{h}_{2}=10<\mathrm{h}_{\mathrm{p}}=20<\mathrm{h}_{\mathrm{C}}=67,
$$

in accordance with Corollary 2.

It is also interesting to check the three different author rankings: in all three cases the set of authors is the same. Let us denote by $r\left(h_{1}, P\right)$ the Spearman rank order correlation coefficient of the comparison of the author ranks according to the author $h$-index $h_{1}$ and according to the number of publications per author. We have $r\left(h_{1}, P\right)=0.854611$ with a corresponding $t$-value of 21.1406463, hence far beyond the classical critical values. We can say that, with a probability of nearly 1 , that there is a positive correlation between the two ranks.

For $r\left(h_{1}, C\right)$, the Spearman rank order correlation coefficient of the comparison of the author ranks according to $\mathrm{h}_{1}$ and according to the number of citations (per author), we have: $r\left(h_{1}, C\right)=0.818131$ with a corresponding t-value of 18.2756527 , showing almost $100 \%$ a positive correlation.

Finally, for $\mathrm{r}(\mathrm{P}, \mathrm{C})$, the Spearman rank order correlation coefficient of the comparison of the author ranks according to the number of publications and according to the number of 
citations, we have $r(P, C)=0.643224$ which is, due to the large value $\mathrm{N}=167$ of number of authors, almost $100 \%$ a positive correlation.

It is clear that a positive correlation between $\mathrm{h}_{1}$ and $\mathrm{P}, \mathrm{h}_{1}$ and $\mathrm{C}$ and $\mathrm{P}$ and $\mathrm{C}$ is logical, but the above results indicate that the correlation is very strong.

\section{$\underline{\text { Remark }}$}

A h-index for a group of authors, different in nature from the ones studied here, is the socalled global h-index, denoted by $\mathrm{h}_{\mathrm{G}}$. The index $\mathrm{h}_{\mathrm{G}}$ is defined as the $\mathrm{h}$-index of the group of authors considered as one meta-author. This index was used in van Raan (2006) in the connection of university research groups in chemistry in the Netherlands.

If we assume that (12) also applies in this global publication-citation setting, then we have that

$$
\mathrm{h}_{\mathrm{G}}=\mathrm{S}^{\frac{1}{\alpha_{2}}}
$$

where $S$ denotes the total number of papers (of all authors). Since clearly $\mathrm{S}^{3} \mathrm{~T}$ (total number of authors), we have, by (16), that

$$
\mathrm{h}_{\mathrm{G}}>\mathrm{h}_{2}
$$

An inequality between $h_{G}$ and the other indices $h_{P}$ and $h_{C}$ is not provable in general due to the appearance of $\alpha_{1}$ in the formulae for $h_{P}$ and $h_{C}$ (and since $\alpha_{1}$ and $\alpha_{2}$ have no a priori relation between each other).

\section{Conclusions}

In this paper we showed that there are, at least, three different ways to calculate an h-index of a group of authors: the successive h-index $h_{2}$, based on the h-indices $h_{1}$ of the authors, the $h$ - 
index $h_{P}$ based on the author-publication ranking and the h-index $h_{C}$ based on the authorcitation ranking.

Concrete formulas for these indices are presented in the framework of Lotkaian informetrics, thereby also proving the inequalities $\mathrm{h}_{2}<\mathrm{h}_{\mathrm{P}}<\mathrm{h}_{\mathrm{C}}$.

A case study of 167 authors in optometry is presented confirming these results and we also show that the three different author rankings (according to $h$-index $h_{1}$, according to number of publications and according to number of citations) have a highly positive correlation value.

It is hard to constitute author data sets as described above. Yet it would be very interesting to have more data of this kind in order to better understand these three indices and to have the results confirmed on the positive correlation between the different rankings.

The first time - we think - that the h-index has been applied to non-citation data was in Liu and Rousseau (2007), where the h-index was applied to library circulation data. To the best of our knowledge, this is the second time that the h-index has been applied to non-citation data ( such as for $\mathrm{h}_{\mathrm{P}}$ ) in a practical case (that the h-index could be defined in a general theoretical IPP setting was already noticed in Egghe and Rousseau (2006)). It is intriguing to explore these simple applications of the h-index to other IPP types. Examples could include calculating the h-index based on papers vs. downloads (replacing citations) or calculating the h-index of websites based on their (in-) links. One could even think of applying the h-index to econometric topics (e.g. calculating the h-index to social groups (e.g. countries, companies, ...) based on their rankings according to wealth or income.

Although it is not clear at the moment which of the h-indices $\left(h_{2}, h_{P}\right.$ or $\left.h_{C}\right)$ should be preferred, it is clear that $\mathrm{h}_{\mathrm{P}}$, the only h-index not based on citation data, is the easiest to calculate since it only uses author publication data. 


\section{$\underline{\text { References }}$}

P. Ball (2005). Index aims for fair ranking of scientists. Nature, 436, 900.

P. Ball (2007). Achievement index climbs the ranks. Nature 448, 737, 2007.

M.G. Banks (2006). An extension of the Hirsch index: indexing scientific topics and compounds. Scientometrics 69(1), 161-168, 2006.

J. Bar-Ilan (2006). H-index for Price medallists revisited. ISSI Newsletter 2(1), 3-5, 2006.

W. Barendse (2007). The strike rate index: a new index for journal quality based on journal size and the h-index of citations. Biomedical Digital Libraries 4(3), 2007. http://www.bio-diglib.com/content/4/1/3

J. Beirlant, W. Glänzel, A. Carbonez and H. Leemans (2007). Scoring research output using statistical quantile plotting. Journal of Informetrics 1(3), 185-192, 2007.

L. Bornmann and H.-D. Daniel (2005). Does the h-index for ranking of scientists really work? Scientometrics, 65(3), 391-392.

L. Bornmann and H.-D. Daniel (2007a). What do we know about the h-index ? Journal of the American Society for Information Science and Technology 58(9), 1381-1385, 2007.

L. Bornmann and H.-D. Daniel (2007b). Convergent validation of peer review decisions using the h-index. Extent of and reasons for type I and type II errors. Journal of Informetrics 1(3), 204-213, 2007.

T. Braun, W. Glänzel and A. Schubert (2005). A Hirsch-type index for journals. The Scientist, 19(22), 8 .

T. Braun, W. Glänzel and A. Schubert (2006). A Hirsch-type index for journals. Scientometrics 69(1), 169-173.

Q.L. Burrell (2007a). Hirsch's h-index: a stochastic model. Journal of Informetrics 1(1), 16$25,2007$.

Q.L. Burrell (2007b). On the h-index, the size of the Hirsch core and Jin's A-index. Journal of Informetrics 1(2), 170-177, 2007.

R. Costas and M. Bordons (2007). The h-index: advantages, limitations and its relation with other bibliometric indicators at the micro level. Journal of Informetrics 1(3), 193203, 2007.

L. Egghe (2005). Power Laws in the Information Process: Lotkaian Informetrics. Elsevier, Oxford (UK). 
L. Egghe (2007a). The influence of transformations on the h-index and the g-index. Preprint.

L. Egghe (2007b). Modelling successive h-indices. Scientometrics, to appear.

L. Egghe (2007c). Dynamic h-index: the Hirsch index in function of time. Journal of the American Society for Information Science and Technology 58(3), 452-454, 2007.

L. Egghe and I.K. Ravichandra Rao (2007). The influence of the query formulation of a topic on its h-index: models and examples of the h-index of N-grams. Preprint.

L. Egghe and R. Rousseau (2006). An informetric model for the Hirsch-index. Scientometrics 69(1), 121-129.

W. Glänzel (2006a). On the opportunities and limitations of the h-index. Science Focus 1(1), 10-11 (in Chinese).

W. Glänzel (2006b). On the h-index - a mathematical approach to a new measure of publication activity and citation impact. Scientometrics 67(2), 315-321.

W. Glänzel and O. Persson (2005). H-index for Price medallists. ISSI Newsletter 1(4), 15-18, 2005.

J.E. Hirsch (2005). An index to quantify an individual's scientific research output.

Proceedings of the National Academy of Sciences of the United States of America $102,16569-16572$.

J.E. Hirsch (2007). Does the h-index have predictive power? arXiv:0708.0646v1 [physics.soc-ph] 6 Aug 2007.

Y. Liu and R. Rousseau (2007). Hirsch-type indices and library management: the case of Tongji university library. In: Proceedings of ISSI 2007. CSIC, Madrid, Spain, 514522.

C.W. Miller (2006). Superiority of the h-index over the impact factor for physics. arXiv:physics/0608183v1 17 Aug 2006.

C. Oppenheim (2007). Using the h-index to rank influential British researchers in information science and librarianship. Journal of the American Society for Information Science and Technology 58(2), 297-301, 2007.

S.B. Popov (2005). A parameter to quantify dynamics of a researcher's scientific activity. arXiv:physics/0508113.

G. Prathap (2006). Hirsch-type indices for ranking institutions' scientific research output. Current Science 91(11), 1439, 2006.

I.K. Ravichandra Rao and R. Rousseau (2007). Empirical series of journal h-indices. Preprint. R. Rousseau (2007). The influence of missing publications on the Hirsch index. Journal of Informetrics 1(1), 2-7, 2007. 
G. Saad (2006). Exploring the h-index at the author and journal levels using bibliometric data of productive consumer scholars and business-related journals respectively. Scientometrics 69(1), 117-120, 2006.

A. Schubert (2007). Successive h-indices. Scientometrics 70(1), 201-205.

A. Schubert and W. Glänzel (2007). A systematic analysis of Hirsch-type indices for journals. Journal of Informetrics 1(3), 179-184, 2007.

The STIMULATE6 Group (2007). The Hirsch index applied to topics of interest to developing countries. First Monday 12(2), 2007. http://www.firstmonday.org/issues/issues/issues12 2/stimulate/

A.F.J. van Raan (2006). Comparison of the Hirsch-index with standard bibliometric indicators and with peer judgement for 147 chemistry research groups. Scientometrics 67(3), 491-502.

J.K. Vanclay (2007). On the robustness of the h-index. Journal of the American Society for Information Science and Technology 58(10), 1547-1550, 2007.

P. Vinkler (2007). Eminence of scientists in the light of the h-index and other scientometric indicators. Journal of Information Science 33(4), 481-491, 2007.

J. Wan, P. Hua and R. Rousseau (2007). The pure h-index: calculating an author's h-index by taking co-authors into account. Preprint. 


\section{$\underline{\text { Appendix }}$}

Table 1. Ranks according to $h$-index values, number of papers and number of citations

\begin{tabular}{|c|c|c|c|c|c|c|c|}
\hline $\begin{array}{c}\text { author } \\
\text { code }\end{array}$ & rank-h & rank-p & rank-c & $\begin{array}{c}\text { author } \\
\text { code }\end{array}$ & rank-h & rank-p & rank-c \\
\hline & & & & a43 & 91 & 91 & 118 \\
\hline a1 & 123 & 49 & 129 & a44 & 47 & 67 & 73 \\
\hline a2 & 5 & 17 & 1 & a45 & 92 & 129 & 110 \\
\hline a3 & 2 & 3 & 4 & a46 & 137 & 130 & 138 \\
\hline a4 & 86 & 74 & 102 & a47 & 93 & 106 & 68 \\
\hline a5 & 14 & 5 & 36 & a48 & 94 & 78 & 125 \\
\hline a6 & 124 & 146 & 95 & a49 & 164 & 154 & 164 \\
\hline a7 & 15 & 26 & 9 & a50 & 138 & 107 & 146 \\
\hline a8 & 125 & 123 & 141 & a51 & 95 & 108 & 120 \\
\hline a9 & 87 & 124 & 58 & a52 & 67 & 21 & 94 \\
\hline a10 & 126 & 102 & 142 & a53 & 96 & 79 & 82 \\
\hline a11 & 34 & 43 & 12 & a54 & 139 & 131 & 147 \\
\hline a12 & 28 & 27 & 49 & a55 & 68 & 52 & 119 \\
\hline a13 & 88 & 125 & 124 & a56 & 97 & 80 & 132 \\
\hline a14 & 127 & 147 & 96 & a57 & 98 & 92 & 100 \\
\hline a15 & 41 & 75 & 14 & a58 & 140 & 155 & 158 \\
\hline a16 & 42 & 50 & 3 & a59 & 69 & 93 & 27 \\
\hline a17 & 128 & 103 & 130 & a60 & 37 & 45 & 63 \\
\hline a18 & 129 & 104 & 99 & a61 & 21 & 39 & 6 \\
\hline a19 & 130 & 148 & 88 & a62 & 99 & 109 & 69 \\
\hline a20 & 20 & 36 & 34 & a63 & 70 & 94 & 89 \\
\hline a21 & 64 & 88 & 57 & a64 & 71 & 11 & 92 \\
\hline a22 & 131 & 149 & 54 & a65 & 72 & 110 & 107 \\
\hline a23 & 162 & 126 & 162 & a66 & 48 & 46 & 42 \\
\hline a24 & 8 & 9 & 17 & $a 67$ & 100 & 82 & 111 \\
\hline a25 & 43 & 89 & 65 & a68 & 141 & 156 & 133 \\
\hline a26 & 163 & 150 & 163 & a69 & 101 & 132 & 80 \\
\hline a27 & 35 & 51 & 52 & a70 & 142 & 111 & 159 \\
\hline a28 & 89 & 105 & 143 & a71 & 102 & 133 & 109 \\
\hline a29 & 44 & 90 & 30 & a72 & 29 & 59 & 19 \\
\hline a30 & 45 & 44 & 5 & a73 & 30 & 68 & 32 \\
\hline a31 & 26 & 28 & 51 & a74 & 73 & 112 & 47 \\
\hline a32 & 65 & 65 & 114 & a75 & 143 & 134 & 116 \\
\hline a33 & 132 & 127 & 144 & a76 & 38 & 82 & 15 \\
\hline a34 & 46 & 76 & 71 & a77 & 103 & 135 & 74 \\
\hline a35 & 33 & 151 & 131 & a78 & 144 & 157 & 77 \\
\hline a36 & 66 & 66 & 97 & a79 & 49 & 22 & 79 \\
\hline a37 & 134 & 152 & 145 & a80 & 145 & 136 & 148 \\
\hline a38 & 135 & 128 & 152 & a81 & 104 & 53 & 121 \\
\hline a39 & 16 & 20 & 35 & a82 & 146 & 137 & 87 \\
\hline a40 & 4 & 5 & 8 & a83 & 22 & 37 & 16 \\
\hline a41 & 136 & 153 & 155 & a84 & 105 & 113 & 134 \\
\hline a42 & 90 & 77 & 115 & a85 & 106 & 114 & 117 \\
\hline
\end{tabular}




\begin{tabular}{|c|c|c|c|c|c|c|c|}
\hline a86 & 74 & 95 & 93 & a127 & 156 & 161 & 98 \\
\hline a87 & 23 & 40 & 21 & a128 & 115 & 61 & 140 \\
\hline a88 & 75 & 60 & 72 & a129 & 116 & 85 & 126 \\
\hline a89 & 147 & 158 & 157 & a130 & 13 & 24 & 18 \\
\hline a90 & 107 & 138 & 112 & a131 & 152 & 116 & 160 \\
\hline a91 & 12 & 18 & 33 & a132 & 153 & 162 & 150 \\
\hline a92 & 50 & 41 & 82 & a133 & 117 & 117 & 127 \\
\hline a93 & 51 & 69 & 64 & a134 & 25 & 34 & 26 \\
\hline a94 & 52 & 30 & 75 & a135 & 7 & 19 & 10 \\
\hline a95 & 17 & 7 & 31 & a136 & 81 & 54 & 103 \\
\hline a96 & 24 & 13 & 50 & a137 & 154 & 163 & 78 \\
\hline a97 & 18 & 6 & 28 & a138 & 57 & 62 & 22 \\
\hline a98 & 76 & 83 & 83 & a139 & 19 & 14 & 44 \\
\hline a99 & 1 & 2 & 2 & a140 & 26 & 35 & 55 \\
\hline a100 & 148 & 139 & 149 & a141 & 155 & 143 & 161 \\
\hline a101 & 108 & 96 & 101 & a142 & 118 & 100 & 106 \\
\hline a102 & 9 & 8 & 23 & a143 & 82 & 118 & 48 \\
\hline a103 & 109 & 140 & 86 & a144 & 83 & 119 & 108 \\
\hline a104 & 77 & 70 & 139 & a145 & 58 & 63 & 56 \\
\hline a105 & 149 & 159 & 153 & a146 & 156 & 120 & 151 \\
\hline a106 & 53 & 15 & 84 & a147 & 119 & 101 & 128 \\
\hline a107 & 110 & 47 & 135 & a148 & 59 & 55 & 85 \\
\hline a108 & 3 & 1 & 7 & a149 & 39 & 56 & 45 \\
\hline a109 & 78 & 32 & 105 & a150 & 40 & 57 & 29 \\
\hline a110 & 6 & 4 & 24 & a151 & 84 & 86 & 41 \\
\hline a111 & 111 & 84 & 61 & a152 & 60 & 87 & 46 \\
\hline a112 & 165 & 97 & 165 & a153 & 157 & 164 & 156 \\
\hline a113 & 54 & 48 & 76 & a154 & 61 & 72 & 70 \\
\hline a114 & 112 & 141 & 39 & a155 & 167 & 165 & 167 \\
\hline a115 & 55 & 38 & 67 & a156 & 158 & 144 & 104 \\
\hline a116 & 56 & 31 & 91 & a157 & 11 & 29 & 11 \\
\hline a117 & 113 & 98 & 122 & a158 & 159 & 166 & 113 \\
\hline a118 & 114 & 142 & 123 & a159 & 120 & 73 & 136 \\
\hline a119 & 31 & 71 & 40 & a160 & 27 & 33 & 20 \\
\hline a120 & 150 & 99 & 154 & a161 & 160 & 167 & 38 \\
\hline a121 & 166 & 160 & 166 & a162 & 62 & 25 & 66 \\
\hline a122 & 32 & 42 & 60 & a163 & 85 & 88 & 37 \\
\hline a123 & 79 & 115 & 53 & a164 & 121 & 145 & 59 \\
\hline a124 & 10 & 16 & 13 & a165 & 63 & 64 & 90 \\
\hline a125 & 80 & 23 & 43 & a166 & 161 & 121 & 137 \\
\hline a126 & 33 & 12 & 25 & a167 & 122 & 122 & 62 \\
\hline
\end{tabular}

